\title{
Central sleep apnoea in congestive heart failure
}

\author{
Atul Malhotra*, Susheel Patil, Scott Sands, and Najib Ayas \\ Pulmonary, Critical Care and Sleep Medicine Division, University of California, San Diego, CA \\ 92037, USA (AM); Johns Hopkins University, Baltimore, MD, USA (SP); Harvard Medical School, \\ Boston, MA, USA (SS); and University of British Columbia, Vancouver, BC, Canada (NA)
}

\begin{abstract}
Abnormal breathing during sleep is common in congestive heart failure. In aggregate, the data suggest that roughly a third of patients with systolic heart failure have obstructive sleep apnoea, a third have central sleep apnoea, and another third have normal breathing. ${ }^{1,2}$ Because the pathophysiology of obstructive and central apnoea are often similar, the term sleep disordered breathing is commonly used to refer collectively to these breathing abnormalities. ${ }^{3-5}$ Cheyne Stokes Respiration (CSR), a form of central sleep apnoea (CSA), has received recent attention due to the fi ndings of the SERVE-HF study ${ }^{6}$ sponsored by ResMed, which showed no improvement in all-cause mortality and an unexpected increase in cardiovascular mortality in patients who received adaptive servo ventilation (ASV) technology compared with controls.
\end{abstract}

ASV is a mode of bilevel positive airway pressure that acts dynamically to maintain regular ventilation by providing increased pressure support (inspiratory minus expiratory pressure) during apnoeas or hypopnoeas and reduced pressure support otherwise.

More than 1300 patients with chronic heart failure, reduced left ventricular ejection fraction (EF $\$ 45 \%$ ), and predominant CSA were randomly assigned to receive either ASV or usual medical care. ${ }^{7,8}$ For this at-risk population there was an increased risk of cardiovascular death (primarily sudden death) compared with controls (absolute annual risk: 10\% in the ASV group vs $7.5 \%$ in the control group). Because details remain sparse, we provide speculation regarding mechanisms that could explain these fi ndings.

First, the deleterious effects of ASV could be spurious. Because the fi ndings of the trial were unexpected, the reproducibility of the fi ndings needs to be challenged and thus further work is still needed. For example, imbalances in baseline characteristics could contribute to the observed outcomes.

The second possibility is that ASV could have direct cardiovascular effects. For example, positive airway pressure is known to reduce cardiac preload. Thus, in theory, patients given ASV might have had a drop in cardiac output with ASV therapy (particularly those with hypovolaemia and low filling pressures). Excessive levels of positive airway pressure could reduce cardiac preload and result in sympathoexcitation. Sustained sympathoexcitation could subsequently trigger arrhy thmias, platelet activation, or plaque rupture. This

*amalhotra@ucsd.edu.

NA reports personal fees from RHS Medical, outside of the submitted work. The other authors declare no competing interests. 
hypothesis could potentially be explored in the SERVE-HF study through interrogation of patients who had automatic implantable cardioverter defibrillators. Posi tive airway pressure can also reduce cardiac afterload, thereby increasing cardiac output in those who are hypervolaemic. ${ }^{9}$ Such an increase in forward flow could increase renal perfusion and yield diuresis with an associated electrolyte abnormality. Hypokalaemia and hypomagnesaemia could both trigger ventricular arrhythmias if appropriate repletion were not provided in a timely manner.

There is no evidence that continuous positive airway pressure (CPAP) per se, as distinct from dynamically applied pressure support of ASV, has deleterious effects on cardiovascular mortality. Notably, there was no CPAP group in the SERVE-HF trial. The fi ndings from the previous (smaller) CANPAP trial examining CPAP in heart failure showed no systematic increase in mortality. ${ }^{10}$ However, the possibility that the specifi c cyclic positive pressure behaviour of ASV could have deleterious cardiovascular effects needs to be considered. When ASV suppresses central sleep apnoea events, positive pressure swings are applied predominantly during the apnoea-hypopnoea phase. ${ }^{7}$ Fluctuations in intrathoracic pressure as a result of variable pressure support might have cardiovascular effects that are deleterious. ${ }^{11}$

A minimum pressure support (default $3 \mathrm{~cm} \mathrm{H}_{2} \mathrm{O}$ ) is often used with ASV and high respiratory rates can also be noted. Thus, there is the possibility of nocturnal hypocapnia with respiratory alkalosis (and ensuing hypokalaemia). Such conditions might trigger arrhythmias in some patients, providing at least a theoretical basis underlying sudden death. ${ }^{12}$ By contrast, available evidence suggests that arterial $\mathrm{PCO}_{2}$ is increased with ASV (presumably due to the removal of the hyperventilatory pattern of CSA) when provided on appropriate settings.

The final possibility is that ASV could have indirect effects (eg, if patients with ASV were uncomfortable, they could have reduced adherence to prescribed medications, which could worsen outcomes). Conversely, some patients have anecdotally experienced major symptomatic improvements with treatment of apnoea leading to excessive activity and sudden death. Suppression of CSR and associated dyspnoea might also remove an important impetus for necessary medical attention. SERVE-HF data on medication adjustments after randomisation may shed light on these possibilities. Lastly, Naughton ${ }^{13}$ hypothesised that CSA has physiological advantages; although unproven, elimination of CSA might have risks.

Questions remain regarding how existing patients should be managed, but clinical experience with ASV withdrawal is limited. First, many patients receive ASV for other diseases including heart failure with preserved ejection fraction, narcotic-induced central apnoea, and treatment emergent central apnoea (so-called complex apnoea). ${ }^{14}$ We believe there is no compelling reason to withdraw ASV from any of these patients at present since the population studied in SERVE-HF consisted primarily of patients with severe heart failure with reduced ejection fraction and predominant CSA. Second, the new trial data suggest that these patients should strongly consider withdrawal from ASV therapy; however, abrupt withdrawal of ASV also carries risks. A history and physical examination will help in 
guiding the physician-patient conversation since optimisation of medical therapy would be recommended before any withdrawal of positive airway pressure therapy, and individu als should be followed closely thereafter. One approach to ASV withdrawal would be to transition patients to CPAP, or use adjunctive therapies to stabilise breathing and improve nocturnal desaturation (oxygen or acetazolamide).${ }^{15}$ No clinical outcome data are available regarding these transition approaches. We recommend that these decisions be based on patient preferences, patient response to therapy, and overall prognosis, taking quality of life into careful consideration. Finally, since SERVE-HF focused on CSA, the question remains whether patients with predominantly obstructive sleep apnoea and congestive heart failure should continue on ASV or be switched to CPAP. We would favour switching such patients to CPAP, recognising that most obstructive breathing abnormalities and some central abnormalities will resolve with this approach.

Substantial progress has been made in our understanding of the importance of sleep and sleep disorders in the context of cardiovascular disease. We strongly support further investigation and view the new results not as a disappointment but rather a call for intensifying our research efforts in this area.

\section{References}

1. MacDonald M, Fang J, Pittman SD, White DP, Malhotra A. The current prevalence of sleep disordered breathing in congestive heart failure patients treated with beta-blockers. J Clin Sleep Med. 2008; 4:38-42. [PubMed: 18350960]

2. Javaheri S, Parker TJ, Liming JD, et al. Sleep apnea in 81 ambulatory male patients with stable heart failure. Circulation. 1998; 97:2154-59. [PubMed: 9626176]

3. Younes M, Ostrowski M, Thompson W, Leslie C, Shewchuk W. Chemical control stability in patients with obstructive sleep apnea. Am J Respir Crit Care Med. 2001; 163:1181-90. [PubMed: 11316657]

4. Jordan AS, McSharry DG, Malhotra A. Adult obstructive sleep apnoea. Lancet. 2014; 383:736-47. [PubMed: 23910433]

5. Malhotra A, Orr JE, Owens RL. On the cutting edge of obstructive sleep apnoea: where next? Lancet Respir Med. 2015; 3:397-403. [PubMed: 25887980]

6. ResMed. ResMed provides update on phase IV SERVE-HF study of adaptive servo-ventilation (ASV) therapy in central sleep apnea and chronic heart failure. San Diego, CA: May 13. 2015 http:// www.resmed.com/us/en/ consumer/newsandinformation/news-releases/2015/resmed-providesupdate-on-phase-iv-serve-hf-study-of-adaptive-servo-ventilation-therapy.html [May 13, 2015]

7. Sharma BK, Bakker JP, McSharry DG, Desai AS, Javaheri S, Malhotra A. Adaptive servoventilation for treatment of sleep-disordered breathing in heart failure: A systematic review and meta-analysis. Chest. 2012; 142:1211-21. [PubMed: 22722232]

8. Cowie MR, Woehrle H, Wegscheider K, et al. Rationale and design of the SERVE-HF study: treatment of sleep-disordered breathing with predominant central sleep apnoea with adaptive servoventilation in patients with chronic heart failure. Eur J Heart Fail. 2013; 15:937-43. [PubMed: 23535165]

9. Fessler H, Brower R, Wise R, Permutt S. Mechanism of reduced LV afterload by systolic and diastolic positive pleural pressure. J Appl Physiol. 1988; 65:1244-50. [PubMed: 3053581]

10. Bradley TD, Logan AG, Kimoff RJ, et al. Continuous positive airway pressure for central sleep apnea and heart failure. N Engl J Med. 2005; 353:2025-33. [PubMed: 16282177]

11. Patruno V, Aiolfi S, Costantino G, et al. Fixed and autoadjusting continuous positive airway pressure treatments are not similar in reducing cardiovascular risk factors in patients with obstructive sleep apnea. Chest. 2007; 131:1393-99. [PubMed: 17494789]

12. Laffey JG, Kavanagh BP. Hypocapnia. N Engl J Med. 2002; 347:43-53. [PubMed: 12097540] 
13. Naughton MT. Cheyne-Stokes respiration: friend or foe? Thorax. 2012; 67:357-60. [PubMed: 22318163]

14. Javaheri S, Malik A, Smith J, Chung E. Adaptive pressure support servoventilation: a novel treatment for sleep apnea associated with use of opioids. J Clin Sleep Med. 2008; 4:305-10. [PubMed: 18763420]

15. Edwards BA, Connolly JG, Campana LM, et al. Acetazolamide attenuates the ventilatory response to arousal in patients with obstructive sleep apnea. Sleep. 2013; 36:281-85. [PubMed: 23372276] 